Nuclear Physics A461 (1987) 305c - 316c

North-Holland, Amsterdam

LATTICE QCD AT FINITE DENSITY

\title{
Frithjof KARSCH*
}

Department of Physics, University of Illinois at Urbana-Champaign, 1110 West Green Street, Urbana, I11inois 6180 ?

We discuss the behavior of the zero temperature limit of lattice field theories with finite chemical potential. The finite chemical potential lattice formalism is applied to the free fermion theory and the Gross-Neveu model where results can be compared with analytic solutions. Problems occuring in the application of this formalism in numerfcal simulations of lattice $\mathrm{QCD}$ are discussed.

\section{INTRODUCTION}

During the recent years we have seen that Monte Carlo simulations of QCD at finite temperature $\mathbf{T}$ can provide detailed quantitative information on the phase structure of QCD and the transition to a quark-gluon plasma at high temperatures. Evidence for the existence of a deconfining phase transition has been found in the pure gauge sector and the existence of a chiral transition in the presence of light dynamical fermions has been established numerically ${ }^{1}$.

In particular in view of future heavy ion experiments which can probe hadronic matter at high densities ${ }^{2}$ it is of great interest to incorporate a non-vanishing chemical potential $\mu$ in these calculations. This would allow to study the phase diagram of QCD in the whole temperature - baryon number density plane. However, at least for the most interesting case of SU(3) gauge theory with dynamical fermions, a numerical analysis at $\mu \neq 0$ is far more complicated than at $\mu=0$. The fermion determinant turns out to be complex. Thus a probability interpretation of the Euclidean path integral is no longer possible and the application of standard simulation techniques is ruled out. It has been suggested to deal with this situation by tak1ng the complex part of the action into the expectation values ${ }^{3}$ or using complex Langevin algorithms ${ }^{4}$. These techniques are, however, still fairly unexplored and at present it is not clear whether they can be used in finite chemical potential calculations ${ }^{5}$. Therefore, the numerical analysis of lattice QCD at $\mu \neq 0$ mainly has been restricted to the quenched sector 6,7 .

\footnotetext{
* address after September 1, 1986: CERN, Theory Division, 1211 Geneve 23, Switzerland.
} 
In this talk we want to discuss some problems arising from such an analysis of the $\mathrm{T}+0$ limit of QCD at non-vanishing chemical potentia1 $\mu$. In particular we will address the question of how far the lattice calculations are able to reproduce the threshold effects related to the discontinuous fermi distribution function at zero temperature. In a theory with massive fermions only the vacuum states will be occupied in the $\mathrm{T} \rightarrow 0$ limit unless the chemical potential is large enough to raise the fermi energy above the energy level of the lowest state with non-vanishing fermion number. For QCD we expect this threshold value $\mu_{0}$ to be given by one third of the nucleon mass, if we assume that there are no stable exotic multiquark or multi-flavor states. The numerical calculations ${ }^{7}$, however, indicate a threshold value given by one half the pion mass. Contrary to our expectations this would indicate that there exist light baryonic states in the QCD spectrum. These problems may be due to a failure of the numerical approximations made ${ }^{8}$ or may even indicate the existence of exotic states in the strong coupling regime. We will present the Monte Carlo data leading to these conclusions in section 4 and discuss the approximations involved. In section 3 we will discuss the low temperature limit of the Gross-Neveu model. This allows to test the finite chemical potential formalism in a non-trivial interacting model which has some similarities with QCD. The Gross-Neveu model has the advantage that unlike QCD it has a real fermion determinant. The effect of dynamical fermions thus can easily be incorporated in the numerical calculations. In addition we can compare the numerical simulations with known continuum results ${ }^{9,10}$.

In this talk we will concentrate on the question in how far the lattice models at $\mathrm{T}=0$ can handle the discontinuous fermi distribution function in the zero temperature limit and want to point out the relation of the associated threshold value for the chemical potential to the mass spectrum of the theory.

Before discussing this issue for interacting lattice models let us illustrate the problem we are addressing in the case of a simple free fermi gas on the lattice.

\section{THE FREE FERMI GAS ON THE LATTICE}

In the case of a free fermionic theory we can study the behavior of exact solutions both in the continum and on the lattice. In the zero temperature limit the fermi distribution function degenerates to a step function $\theta(\mu-m)$ and the partition function becomes 


$$
\begin{aligned}
& \Omega_{0} \equiv-\frac{1}{V} \lim _{T \rightarrow 0} \ln Z \\
& =\theta(\mu-\mathrm{m})\left\{-\frac{1}{3 \pi^{2}}\left[\frac{1}{4} \mu\left(\mu^{2}-\mathrm{m}^{2}\right)^{1 / 2}\left(\mu^{2}-\frac{5}{2} \mathrm{~m}^{2}\right)+\frac{3}{8} \mathrm{~m}^{4} \ln \left(\frac{\mu+\left(\mu^{2}-\mathrm{m}^{2}\right)^{1 / 2}}{\mathrm{~m}}\right)\right]\right\}
\end{aligned}
$$

Thus a chemical potential larger than the rest mass of the fermions is necessary in order to occupy states above the vacuum which can contribute to the partition function. From Eq. 1 we obtain for the number density $n$

$$
n=\theta(\mu-\mathrm{m})\left\{\frac{1}{3 \pi^{2}}\left(\mu^{2}-\mathrm{m}^{2}\right)^{3 / 2}\right\}
$$

which again reflects the existence of a threshold value $\mu_{0}$

$$
\mu_{o} \equiv \mathrm{m}
$$

which the chemical potential has to exceed in order to create a nonvanishing number density. We now want to discuss how this threshold behavior of the continum theory is realized in the lattice version of the free fermion model. The parition function on a 4 -dimensional Euclidean lattice is given by

$$
z=\int \frac{\pi}{x} d x_{x} d \vec{\chi}_{x} e^{-S} F
$$

where the action for fermions of mass $m$ is given by

$$
s_{F}=m \sum_{x} \bar{x}_{x} x_{x}+\sum_{x, \nu} \bar{x}_{x} D_{x y}^{\nu} x_{y}
$$

and

$$
\begin{aligned}
& D_{x y}^{i}=\frac{1}{2} n_{i}(x)\left[\delta_{y, x+i}-\delta_{y, x-i}\right] i=1,2,3 \\
& D_{x y}^{o}=\frac{1}{2}\left[e^{\mu} \delta_{y, x+o}-e^{-\mu} \delta_{y, x-0}\right]
\end{aligned}
$$

Note that the chemical potential has been introduced in the imaginary time direction of the lattice Dirac operator by providing a factor exp $\{\mu\}$ in the forward and exp $\{-\mu\}$ in backward direction $6,11,12$. In Eq. 4 we used staggered fermions to discretize the Dirac operator. It has been checked that in the limit of vanishing lattice spacing this formulation reproduces 
the known continuum results for the $T=0$ theory ${ }^{6,11,13}$. Here we want to show that also at finite lattice spacing a the free fermi model shows the expected threshold behavior in the zero temperature limit. The partition function can be evaluated exactly and we obtain for the number density ${ }^{13}$.

$$
\mathrm{n}=\theta(\mu-E(0)) \int_{-\pi}^{\pi} \frac{\mathrm{d}^{3} \mathrm{p}}{(2 \pi)^{3}} \theta(\mu-E(\overrightarrow{\mathrm{p}}))
$$

with

$$
E(p)=\sinh ^{-1}\left(\sqrt{m^{2}+\sum_{i=1}^{3} \sin ^{2} p_{i}}\right)
$$

Thus the number density stays zero as long as the chemical potential is smaller than

$$
\mu_{0}=\mathrm{E}(0)=\ln \left[\mathrm{m}+\sqrt{1+\mathrm{m}^{2}}\right]
$$

We note that this value agrees with the mass of the free lattice fermion at finite lattice spacing which is defined by the pole of the free fermion lattice propagator at zero momentum. The behavior of the number density is shown in Fig. 1 for several mass values.

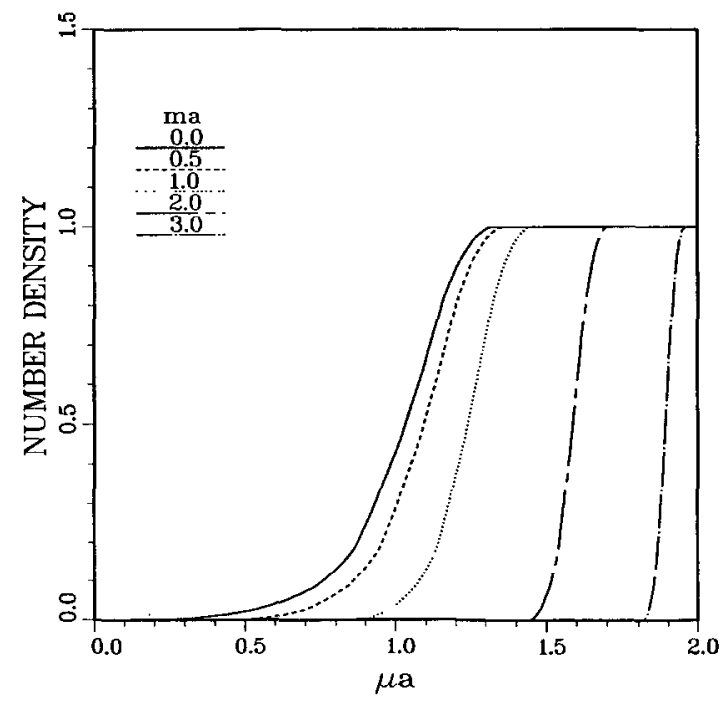

Figure 1: Number density versus chemical potential for the free fermi gas at zero temperature on an infinite lattice. Shown are results for different bare masses as indicated in the figure. 
Thus the free fermion lattice gas model works as expected. In the zero temperature 1imit we find a threshold value for the chemical potential below which all observables agree with the $\mu=0$ values. In particular the number density stays zero for all $\mu<\mu_{0}$. In the following we will discuss the behavior of the Gross-Neveu model at finite $\mu$. This tests the finite chemical potential formalism in the case of a non-trivial interacting fermion model on the lattice and allows to illustrate the relation between the threshold value $\mu_{0}$ and the mass spectrum of the model.

3. THE GROSS-NEVEU MODEL

Many features of the Gross-Neveu model (GNM) ${ }^{9}$ are similar to those expected from QCD. This makes it a particular interesting toy model. The GNM is a 2-dimensional model for $n$ different species of fermions interacting through a four fermion term. It is asymptotically free and has a spontaneously broken (discrete) chiral symmetry. The quartic interaction term can be decomposed into a mass term by introducing a real scalar field $\sigma$ in the Lagrangian. Several lattice versions of this model have been analyzed $^{14}$ which for large values of $n_{f}$ reproduce well known continuum results for the $n_{f} \rightarrow \infty$ limit of this model. We will use a lattice Lagrangian that introduces the scalar field $\sigma$ on plaquettes of the 2-dimensional lattice

$$
\begin{aligned}
& s^{G N}=\sum_{k} \sum_{x, y}\left[\bar{x}_{x}^{(k)} D_{x y}^{o} x_{y}^{(k)}+\bar{x}_{x}^{(k)} D_{x y}^{l} x_{y}^{(k)}\right]+\frac{1}{2 g^{2}} \sum_{x} \sigma_{x}^{2} \\
& +\frac{1}{4} \sum_{x, k}\left[\sigma_{x+o}+\sigma_{x-o}+\sigma_{x+1}+\sigma_{x-1}\right] \bar{x}^{(k)} x_{x}^{(k)}
\end{aligned}
$$

with the partition function given by

$$
Z=\int \prod_{x} d \sigma_{x} \prod_{x, k} d x_{x}^{(k)} d \bar{x}_{x}^{-(k)} e^{-S^{G N}}
$$

In Eq. $9 / 10 \mathrm{k}$ labels the $\mathrm{n}_{\mathrm{f}} / 2$ different fermion flavors (In 2 dimensions the number of flavors gets doubled in the continuum limit). We are interested in the behavior of the lattice model at finite $\mu$ and zero temperature. We want to determine the threshold value for the chemical potential at which states with non-vanishing fermion number start being occupied. This threshold value will be related to the spectrum of the interacting model. In the continuum this spectrum is well known ${ }^{9}$. The fermion sector consists of a heavy fermionic state $\mathrm{m}_{1}$, 


$$
\mathrm{m}_{1}=\sigma_{\mathrm{o}}
$$

and a sequence of bound states whose masses are given by

$$
m_{n}=\sigma_{0} \frac{2 n_{f}}{\pi} \sin \left(n \frac{\pi}{2 n_{f}}\right) \quad, n=1,3, \quad n_{f}-1
$$

with $\mathrm{n}$ being the number of fermions (antifermions) in the bound state and $\sigma_{0}$ denoting the vacuum expectation value of the scalar field $\sigma$. Assuming that at $\mu_{0}$ we create a low density non-interacting gas of particles, we expect to find a threshold value determined by that bound state that leads to the minimal energy per constituent. In the large $n_{f}$ limit, where the above mass relations are valid, we thus would expect to find for the threshold value.

$$
\mu_{0}=\min _{n} \frac{m_{n}}{n}=\frac{2}{\pi} \sigma_{o}
$$

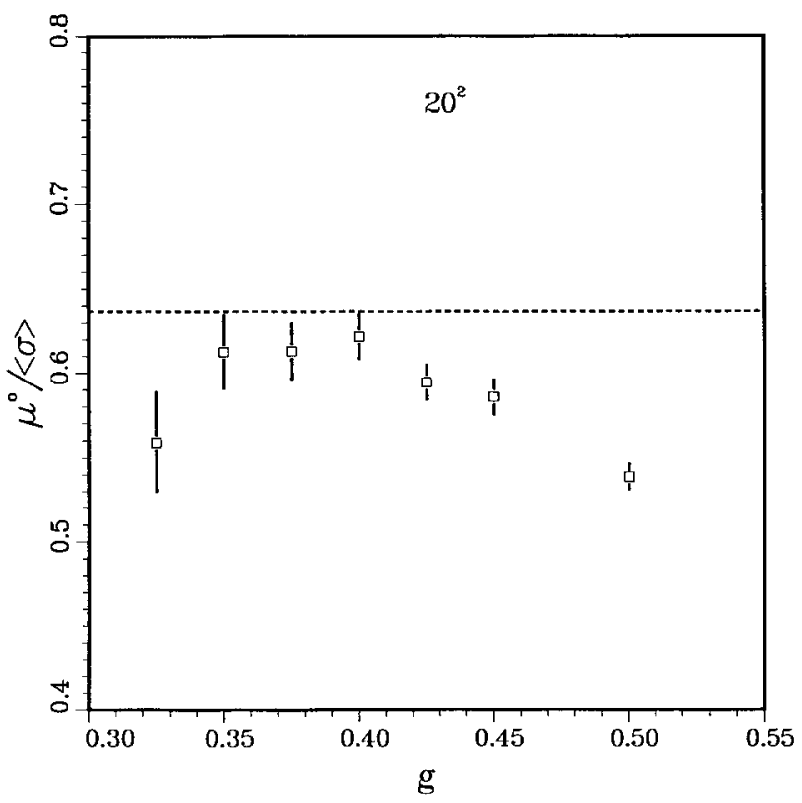

Fig. 2: The threshold value $\mu_{0}$ normalized with the vacuum expectation value of the scalar field $\sigma_{0}=\langle\sigma\rangle$ versus coupling $g$. The data points have been obtained from a measurement of the fermion number density on a $20 \times 20$ latice with $\mathrm{n}_{\mathrm{f}}=12$ flavors of fermions 15 . 
Thus we expect that at $\mu_{o}$ multi-fermion bound states with $n=n_{f}$ fermions are created at rest. We have analyzed this question in a Monte Carlo simulation and determined $\mu_{0}$ from points where the number density starts getting nonzero ${ }^{15}$. The simulations have been performed for the case of $n_{f}=12$ fermion flavors in order to be able to compare numerical results with exact continuum results for the $\mathrm{n}_{\mathrm{f}}+\infty 1 \mathrm{imit}$.

In FIg. 2 we show a measurement of $\mu_{0} / \sigma_{0}$ for various couplings $g$. We see that coming from the strong coupling region the ratio $\mu_{0} / \sigma_{0}$ indeed approaches the expected value $2 / \pi$ in the scaling region. For too small couplings, $1 . e$. $g<0.35$, we again observe deviations from this asymtotic value. This is probably due to finite temperature effects setting in for this small couplings. A detailed discussion of the Gross-Neveu model at fintte $\mu$ and finite temperature is given in Ref. 15 where also the chiral transition is studied in detail ${ }^{10}$. Here we note that also in an interacting fermion model the behavior in the zero temperature limit follows our expectations as far as the onset of thermodynamics is concerned. The value found for $\mu_{0}$ is consistent with the idea that at $\mu_{0}$ we start creating multi-fermion bound states. This can be checked explicitly by analyzing typical configurations contributing to the partition function close to $\mu_{0}$.
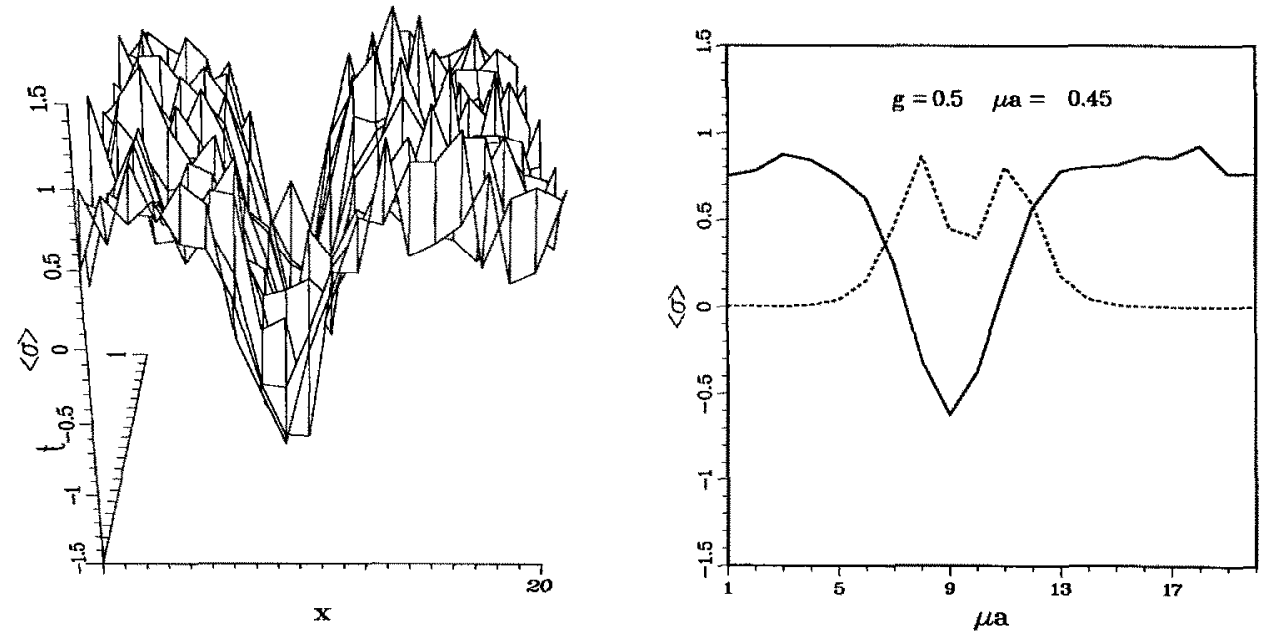

Fig. 3: A typical configuration on the $20 \times 20$ lattice at $g-0.5$ and $\mu=0.45(\mathrm{Fig} \cdot 3 \mathrm{a})$. Fig. $3 \mathrm{~b}$ shows a projection of this configuration on the spatial axis (solid line) and the spatial distribution of the number density divided by $n_{f} / 4$ in this configuration (broken line). 
In Fig. 3a we show a typical configuration at $g=0.5$ generated for $\mu$ slightly above $\mu_{0}$. The configuration clearly shows a kink-antikink which is the wave function for a multi-fermion bound state. In Fig. 3b we show a projection of this state on the spatial axis together with the spatial distribution of the fermion number density. In each edge of the kinkantikink wavefunction we find 6 fermions localized. Thus the state consists of $\mathrm{n}=\mathrm{n}_{\mathrm{f}}=12$ states as expected.

\section{PROBLEMS WITH FINITE DENSITY SIMULATIONS OF LATTICE QCD}

From the analysis of the free fermion model and the Gross-Neveu model we see that the finite density formulation of lattice field theories works well and is sensitive to the mass spectrum of the models. In the case of QCD we thus would expect to find a threshold value $\mu_{0}$ which is related to the creation of the lightest baryonic states, i.e. nucleons. Thus if we assume to create a low density non-interacting nucleon gas ${ }^{+}$at $\mu_{0}$ we expect

$$
\mu_{\mathrm{o}}=\mathrm{m}_{\mathrm{N}} / 3
$$

Unlike in the GNM we do not have a reliable numerical method to deal with the full interacting theory. We thus analyzed a limit where the influence of dynamical fermions is expected to be small and where we can compare numerical results with analytic (mean field) calculations.

In Fig. 4a we show results for the baryon number density for QCD in the strong coupling limit $\left(6 / \mathrm{g}^{2}=0\right)$. These data have been obtained in the quenched approximation ${ }^{7}$. The existence of a threshold value is clearly visible. However, a comparison of the measured values for $\mu_{0}$ with known hadron masses in the strong coupling 1 imit ${ }^{16}$ shows that instead of being related to the nucleon mass, $\mathrm{m}_{N}, \mu_{0}$ is determined by

$$
\begin{aligned}
& \mu_{0}=m_{\pi} \\
& =\frac{1}{2} \ln \left[1+\frac{1}{2}\left(c^{2}-2 d\right)+/\left(c^{2}-2 d\right)+\frac{1}{4}\left(c^{2}-2 d\right)^{2}\right]
\end{aligned}
$$

\footnotetext{
+In the absence of Coulomb repulsion bulk nuclear matter will be stable. Thus we would actually expect to find a somewhat smaller value for $\mu_{0}$ : one third of the nucleon mass minus the binding energy of nuclear matter. A priory there is no reason to expect this shift to be sma11.
} 
with $d=4, c=m+\sqrt{2 d+m^{2}}$ and $m_{\pi}$ being the pion mass. The critical value seems to approach zero as the quark mass is lowered. Similar results hold for the chiral condensate which we show in Fig. 4b.
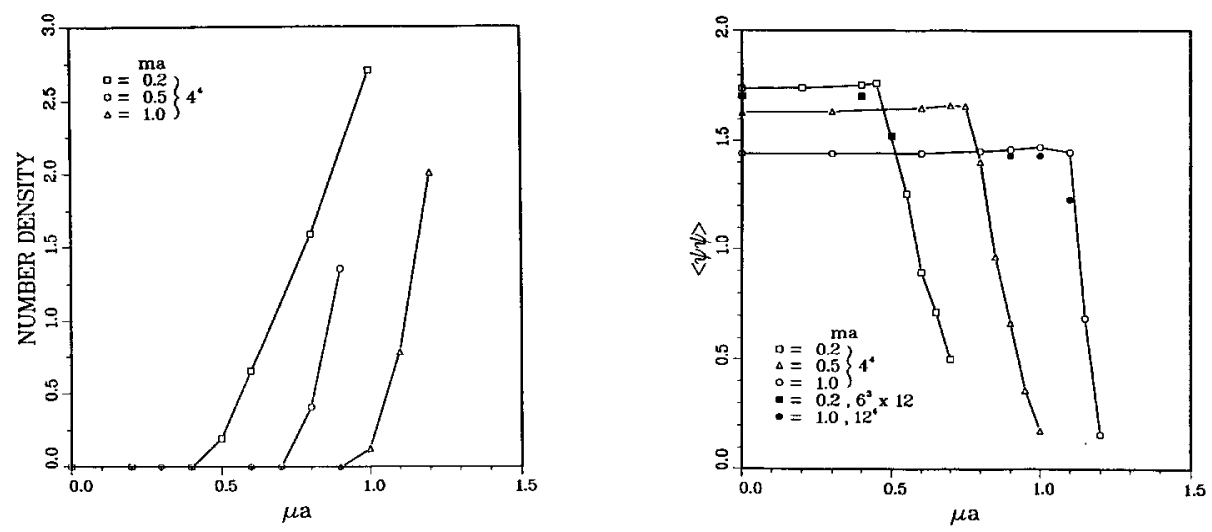

Fig. 4: The baryon number density for the SU(3) gauge theory with staggered fermions of mass ma $=0.2,0.5$ and 1.0 versus chemical potential on a $4^{4}$ lattice (Fig. 4.a). Fig. 4b shows similar results for the chiral condensate $\langle\bar{\chi} \chi\rangle$ on lattices of various sizes ${ }^{7}$.

Some of this data has been obtained on fairly large lattices and using exact matrix inversion routines to calculate the fermion propagator. This seems to rule out that finite size problems or problems related to an insufficent algorithm for quenched calculations are responsibile for the unexpected result manifested by Eq. 15. It has been argued that the problems observed in quenched calculations are entirely due to this approximation ${ }^{8}$ which at present can not be ruled out. We performed, however, some calculations using a complex Langevin algorithm to incorporate dynamical fermions ${ }^{4,17}$. This did not improve the situation for SU(3) although it performed well for a $\mathrm{U}(3)$ theory. It may, however, be that the complex Langevin algorithm which itself is not well founded fails for SU(3) gauge theory and more refined techniques may be necessary to deal correctly with the contribution of dynamical fermions.

Beside the discussed problem of an unexpected small value for $\mu_{0}$ the results shown in Fig. 4 also seem to have undesirable consequences for the pattern of chiral symmetry breaking at non-zero chemical potential ${ }^{7}$. Like in similar calculations for $\operatorname{SU}(2)$ gauge theory ${ }^{18}$ we observe that $\langle\bar{\chi} \chi\rangle$ seems 
to drop to zero as soon as the number density becomes non-zero. This suggests that at finite baryon number density chiral symmetry is restored.

The phase diagram emerging from the analysis of quenched strong coupling QCD at $\mu \neq 0, T=0$ is shown in Fig. 5. There we also show the expected result based on the relation $\mu_{0}=\mathrm{m}_{\mathrm{N}} / 3$, with $\mathrm{m}_{\mathrm{N}}$ being the nucleon mass at strong coupling ${ }^{16}$. Fig. 5 also shows the result obtained from a mean field calculation ${ }^{7}$ which agrees with the numerical data for large quark masses but then approaches a constant value for $\mu_{0}$ in the zero mass limit. The mean field analysis gives a first order phase transition from the vacuum phase (no fermions) to a densely packed state (maximun number of fermions per site). This may be interpreted as a state of bulk nuclear matter (see footnote ${ }^{+}$).

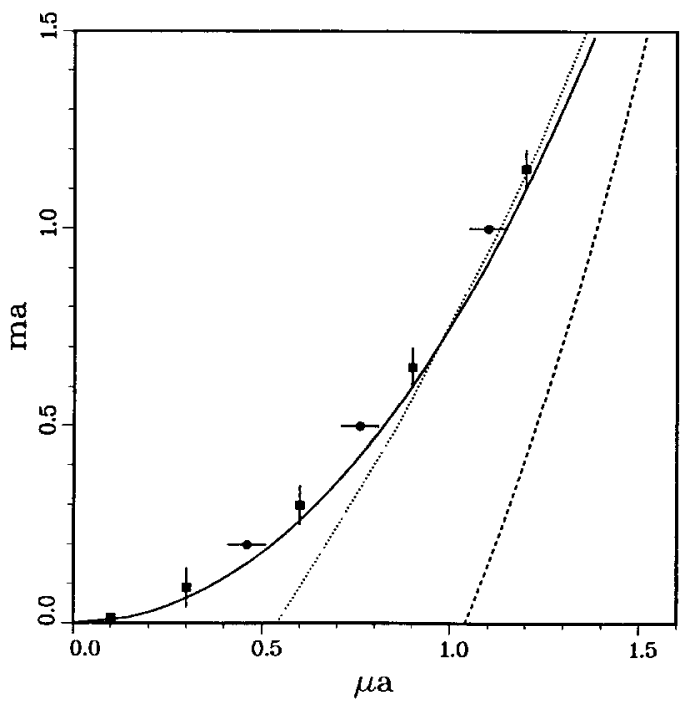

Fig. 5: Phase diagram in the mass-chemical potential plane of the SU(3) gauge theory with staggered fermions in the strong coupling limit. The data points show the threshold value where physical observables start deviating from their $\mu=0$ values. Also shown is the expected critical line corresponding to the baryon threshold (- - ) and the pion threshold (-- - ) which seems to describe the Monte-Carlo data. The dotted curve shows the result of a mean-field calculation discussed in Ref. 7. 


\section{CONCLUSIONS}

We have seen that the formulation of lattice models with non-vanishing chemical potential works well in the case of the free theory and the GrossNeveu model. In these cases the effect of dynamical fermions can be included correctly in the numerical analysis and the lattice calculations could reproduce known continuum results.

In the case of QCD the analysis of strong coupling lattice theory using quenched simulation techniques lead to unexpected results. It may be that these results are due to a failure of the quenched approximation and a correct implementation of dynamical fermions may resolve the present problems. To judge this we require a reliable algortthm that can simulate lattice models with complex actions.

\section{REFERENCES}

1. For a recent review see: F. Karsch, "QCD at Finite Temperature and Baryon Number Density", Illinois Preprint, I11-(TH)-86-\$9, January 1986.

2. J. Cleymans, R. V. Gavai and E. Suhonen, Phys. Rep. 130 (1986) 217.

3. J. Engels and H. Satz, Phys. Lett. 159B (1985) 151.

4. F. Karsch and H. W. Wyld, Phys. Rev. Lett. 55 (1985) 2242.

5. See talk by $J$. Engels at this meeting.

6. J. Kogut, H. Matsuoka, M. Stone, H. W. Wyld, S. Shenker, J. Shigemitsu and D. K. Sinclair, Nuc1. Phys. B225 [FS9] (1983) 93.

7. I. Barbour, N-E. Behilil, E. Dagotto, F. Karsch, A. Moreo, M. Stone and $\mathrm{H}$. W. Wyld, Illinois Preprint ILL-(TH)-86\#23.

8. P. E. Gibbs, University of Glasgow Preprint.

9. D. J. Gross and A. Neveu, Phys. Rev. D10 (1974) 3235; R. F. Dashen, B. Hasslacher and A. Neveu, Phys. Rev. D12 (1974) 2443.

10. For a discussion of the $\mu-T$ phase diagram in the continuum model see: U. Wolff, Phys. Lett. 157B (1985) 303.

11. P. Hasenfratz and F. Karsch, Phys. Lett. 125B (1983) 308.

12. For other formulations see: N. Bilic and R. V. Gavai, Z. Phys. C23 (1984) 204.; R. V. Gavai, Phys. Rev. D32 (1985) 519.

13. H. Matsuoka and M. Stone, Phys. Lett. 136B (1984) 204.

14. Y. Cohen, S. Elitzur and E. Rabinovici, Phys. Lett. 104B (1981) 389 and Nucl. Phys. B220 [FS8] (1983) 102. 
15. F. Karsch, J. Kogut and H. W. Wyld, in preparation.

16. H. K1uberg-Stern, A. More1 and B. Peterson, Nuc1. Phys. B215 [FS7] (1983) 527.

17. G. Paris1, Phys. Lett. 131B (1983) 393; J. Klauder, "Stochast1c Quantization", Lectures given at the XXII Schladming School, March 1983.

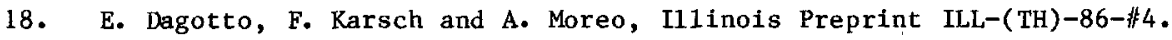

